TJVR 2021; 5 (1): 35-40

Turkish Journal of Veterinary Research

http://www.dergipark.gov.tr/tjvr

e-ISSN: 2602-3695

\title{
Evaluation of the effect of Carvacrol on retinal neovascularization in rats
}

\author{
Abdulgani Kaymaz ${ }^{1}$ Sevilay Erimşah²® \\ ${ }^{1}$ Department of Ophthalmology, Faculty of Medicine, Bolu Abant Izzet Baysal University, Bolu, Turkey \\ 2 Department of Histology and Embryology, Faculty of Medicine, Kocaeli University, Kocaeli, Turkey
}

\begin{abstract}
Objectives: To compare the effect of intraperitoneal administered Carvacrol with bevacizumab in an oxygen-induced retinopathy (OIR) model in rats.

Materials and Methods: Twenty-eight newborn rats were included in the study and the OIR model was created with the $50 / 10 \%$ oxygen style. The study consisted of 4 groups and each rat in the groups received an intraperitoneal injection of $0.01 \mathrm{~mL}$ once on the postnatal $14^{\text {th }}$ day. While the OIR model was not created in Group I (control group), it was created for Groups II, III, and IV. Groups I and II were injected with $0.9 \%$ $\mathrm{NaCl}$ solution, Group III with bevacizumab, and Group IV with carvacrol. The rats were sacrificed on the postnatal $18^{\text {th }}$ day.

Results: Histopathological and immunohistochemical studies showed that the number of retinal vascular endothelial cells (RVECs) and nuclear factor (NF)-kB levels decreased similarly in Group III and Group IV compared to Group II. RVECs values for Group I, Group II, Group III and Group IV were measured as $1.26 \pm 0.80,27.10 \pm 3.63,7.54 \pm 1.38$, and $6.22 \pm 1.22$, respectively and it differed significantly between groups $(\mathrm{p}<0.001)$. Likewise, NF- $\kappa \mathrm{B}$ levels were recorded as $0.61 \pm 0.30,4.36 \pm 0.65,2.68 \pm 0.44$, and $2.85 \pm 0.58$, respectively and it differed significantly between groups $(\mathrm{p}<0.001)$. On the other hand, RVECs and NF- $k B$ levels were similar between Group III, and Group IV (p values were 0.58 and 0.91, respectively).
\end{abstract}

Conclusions: The study demonstrated that carvacrol significantly reduced retinal pathological neovascularizations, RVECs, and NF- $\mathrm{kB}$ levels. Moreover, the observed effects were comparable to those of bevacizumab.

Keywords: Bevacizumab, Carvacrol, Neovascularization, Nuclear factor- $\kappa B$, Oxygen-induced retinopathy, Retinal vascular endothelial cell

\section{INTRODUCTION}

Although neovascularization (NV) is physiologically vital, it is also the main cause of many diseases. Excessive NV's may result in vision loss and blindness in the eye (Ferris et al., 1984). Diabetic retinopathy (DRP), retinopathy of prematurity (ROP), age-related macular degeneration (AMD), and pathological NVs due to retinal vascular occlusions (RVOs) are the major causes of vision loss (Engerman, 1989; Ferris et al., 1984; Green, 1999). In these diseases, as a result of the triggering of inflammatory cytokines, retinal NVs and edema develop, and a decrease in visual acuity occurs (Engerman, 1989; Green, 1999; Klaassenet al., 2013). Although many mediators are blamed for this process, intertwined proinflammatory and anti-inflammatory stages are mentioned (Klaassen et al., 2013). Among the prominent mediators include vascular endothelial growth factor (VEGF) and nuclear factor- $\kappa \mathrm{B}$ (NFкB) (Ascaso et al., 2014). 
The oxygen-induced retinopathy (OIR) model imitates the vaso-occlusive and NV phases of ROP and enables the trial of new drugs to be used for treatment (Smith et al., 1994). In this model, the normal vascular development of the retina and pathological NV formation can also be examined (Smith et al., 1994). Hypoxia occurring in the retina for any reason causes the release of pro-angiogenic factors and the formation of NV (Adamis et al., 1994). NF- $\kappa B$, released and controlled by retinal vascular endothelial cells (RVECs), has an important role in the $\mathrm{NV}$ formation process (Moraiset al., 2009; Zhanget al., 2011). NF- $\kappa B$ indirectly contributes to VEGF production. When inhibited, it inhibits VEGF, thus angiogenesis (Halleet al., 2011; Zhang et al., 2011). In addition, when VEGF is inhibited, NF- $\mathrm{BB}$ and NV formation have been shown to be inhibited (Lopezet al., 2019).

Bevacizumab (Avastin ${ }^{\circledR}$ ), is a monoclonal antibody that inhibits VEGF activity and is increasingly used for the treatment of various proliferative retinopathies (Maguire et al., 2016; Rich et al., 2006). Treatment with anti-VEGF agents (such as aflibercept, ranibizumab, and bevacizumab) prevented the pathological NVs seen in DRP, AMD, and ROP, reduced macular edema, decreased vision loss, and increased visual acuity (Maguire et al., 2016; Talks et al., 2016). In addition, it is a known fact that bevacizumab indirectly reduces NF- $\kappa B$ and NV since it inhibits VEGF (Lopez et al., 2019).

Carvacrol is the most important ingredient in thyme's essential oils (Baser, 1994). Carvacrol, which is widely used in medicine, pharmacy, and agriculture, has been used since ancient times with its medicinal benefits, and new benefits are discovered day by day (Altundağ and Aslım, 2005). Carvacrol has a wide range of therapeutic effects, such as anti-angiogenic, anti-inflammatory, and anti-oxidant actions (Liet al., 2019; Mahmoodi et al., 2019). Studies have shown that carvacrol regulates the balance between pro-inflammatory and antiinflammatory mediators and suppresses the inflammatory response (Liet al., 2019; Mahmoodi et al., 2019). Besides these effects, carvacrol additionally reduces VEGF, NF- $\kappa \mathrm{B}$, and suppresses NV (Bayramoglu et al., 2014; Li et al., 2019; Mahmoodi et al., 2019). It has been reported that due to the ability of Carvacrol to inhibit NF- $\kappa \mathrm{B}$, it can also prevent $\mathrm{NV}$ development and metastasis in cancers (Cui et al., 2015).

Although the anti-angiogenic effect of carvacrol has been studied in many tissues and organs, including cancers, the number of studies on the retinal $\mathrm{NV}$ is quite limited (Bayramoglu et al., 2014; Husseinet al., 2017). Therefore, we planned to investigate the effect of carvacrol administered intraperitoneally in the OIR model on retinal NV and to compare it with the routinely used bevacizumab.

\section{MATERIALS and METHODS}

The present study was carried out in accordance with the ARVO Statement on the Use of Animals in Ophthalmic and Vision Research guidelines. The study was approved by the Bolu Abant İzzet Baysal University Experimental Animal Studies Ethics Committee as the local ethics committee (No: 2021/02).

Twenty-eight newborn Sprague Dawley rats were included in the study. To establish the OIR model, the newborn pups were placed with their mothers in an oxygen-regulated environment Oxycycler (Biospherix Ltd., Lacona, NY) within 4 hours after birth, where they were exposed to $50 \%$ oxygen for 24 hours followed by $10 \%$ oxygen for 24 hours (Pennet al., 1994). A 12-hour light: 12-hour dark environment was created in the care room with a temperature of $22-24^{\circ} \mathrm{C}$ and relative humidity of $45-$ $60 \%$. Food and beverage restrictions were not applied. This 50/10\% oxygen cycle was repeated seven times until the 14th postnatal day (PND). Oxygen and carbon dioxide levels were monitored daily and calibrated as needed. In PND 14, pups were placed in a room with ambient air for 4 days.

A total of four groups were formed, including seven rats in each group. Ip injections were administered to the rats only once on PND 14 when they were taken to the care room.

Group I: $0.01 \mathrm{ml}$ ip $0.9 \% \mathrm{NaCl}$ solution was administered without creating the OIR model (control group).

Group II: OIR model was created and $0.01 \mathrm{ml}$ ip $0.9 \% \mathrm{NaCl}$ solution was administered (Untreated OIR group).

Group III: OIR model was created and $0.01 \mathrm{ml}(2.5$ $\mathrm{mg} / \mathrm{kg}$ ) ip bevacizumab (Altuzan, Roche, Istanbul, Turkey) treatment was applied (Acun et al., 2018).

Group IV: OIR model was created and $0.01 \mathrm{ml}$ (73 $\mathrm{mg} / \mathrm{kg}$ ) ip carvacrol (Sigma-Aldrich, St. Louis, USA) treatment was applied (Şen et al., 2014).

In this OIR model, the best NV formation is assumed to be 18-20th PNDs. Therefore, after anesthesia was provided with intramuscular ketamine/xylazine, approximately $5 \mathrm{ml}$ of blood was taken from all pups by intracardiac puncture, 
and sacrification was performed in PND 18 and their right eyes were enucleated. Tissues were taken for histopathological and immunohistochemical analyses.

\section{Quantification of retinal vascular endothelial cells and immunohistochemistry}

The paraffin-embedded eyes were fixed in 10\% neutral formalin and paraffin-embedded serial sections $(4 \mu \mathrm{m})$ of eye tissue were obtained sagittally then stained with Hematoksilen and Eosin (H\&E). The nuclei of retinal vascular endothelial cells (RVEC) on the vitreal side of the retinal inner limiting membrane (ILM) were counted in ten sections of each eye at $x 40$ objective as previously described (Şen et al., 2014). The mean number of the endothelial cell nuclei of each eye was counted and calculated by two independent reviewers blind to the experiment.

Immunohistochemistry was performed using a biotin-streptavidin HRP detection kit (ab93697; Abcam, Cambridge, UK). Formalin-fixed and paraffin-embedded eye tissue sections $(4 \mu \mathrm{m})$ were deparaffinized in xylene, and rehydrated in graded ethanol series. Citrate buffer was used as antigen retrival. Endogenous peroxidase was blocked with $\mathrm{H} 2 \mathrm{O} 2(3 \%)$ in methanol. The sections were treated by a blocking serum (Histostatin plus kit broadspectrum; Invitrogen, California, USA). The sections were then incubated with anti-NF- $\kappa$ B P65 antibody ( $a b$ 16502), and the biotinylated secondary antibodies (Mouse and Rabbit Specific HRP Plus (ABC) Detection IHC kit (ab93697; Abcam) at room temperature. 3,3-diaminobenzidine (DAB kit 882014, Invitrogen, California, USA) was used to visualize the peroxide complex. Finally, the sections were counterstained with Mayer's hematoxylin (Invitrogen, California, USA), dehydrated, and mounted with Entellan. The images were observed with a light microscope and photographed. Each section was graded as: no expression, 0 ; weak, 1; moderate, 2; strong, 3; and very strong expression, 4 . The percentage of positive cells was defined as 0 , $<5 \% ; 1,6 \%$ to $15 \% ; 2,16 \%$ to $50 \% ; 3,51 \%$ to $80 \%$; and $4,>80 \%$ positive cells (Figure 2) (Gocmezet al., 2015). The mean value was determined by analysing 3 retinal sections of each rat.

\section{Statistical Analysis}

Data analyzes were performed using the SPSS statistical software package, version 25.0 (SPSS Inc., Chicago, IL, USA). Data are presented as mean \pm standard deviations (SDs) for each data set. Statistical significance was accepted as $\mathrm{p}<0.05$.
Statistical analysis of the data was performed using one-way analysis of variance test and post hoc analysis after the homogeneity and normality of sample distribution was confirmed.

\section{RESULTS}

\section{Quantitative analysis of RVEC with H\&E staining}

The nuclei of retinal vascular endotelial cells were counted, and quantified to confirm the effects of carvacrol on NV (Figure 1). Group I have a histology of normal retina (Figure 1A). In Group II, many new blood vessels growing in the vitreous humor (Figure 1B, black arrow) and enlarged intraretinal vessel profiles were seen in the ILM, resulting as a characteristic of OIR. In Group IV, there was a reduced number of RVEC and vascular tufts on the vitreal side of the retinal ILM (Figure 1D) similar to Group III (Figure 1C). RVECs values for Group I, Group II, Group III, and Group IV were measured as $1.26 \pm 0.80,27.10 \pm 3.63,7.54 \pm 1.38$, and $6.22 \pm 1.22$, respectively and it differed significantly between groups $(\mathrm{p}<0.001)$.

\section{Expressions of NF- $\kappa B$}

Immunochemistry was also performed to investigate expression levels of NF- $\kappa \mathrm{B}$ (Figure 2). In Group I, NF-kB expression levels were weakly seen only in the GCL layer (Figure 2A). However, in Group II, brown stained nuclei were strongly detected in the GCL, INL, IPL and OPL (Figure 2B). The expression levels of NF-kB in Group III and Group IV were lower in the retinal layers with reduced neovascularization on the vitreal side of the retinal inner limiting membrane (ILM) compered to Group II (Figure $2 \mathrm{C}$ and Figure 2D). NF-kB levels for Group I, Group II, Group III, and Group IV were recorded as $0.61 \pm 0.30,4.36 \pm 0.65$, $2.68 \pm 0.44$, and $2.85 \pm 0.58$, respectively and it differed significantly between groups ( $\mathrm{p}<0.001)$.

When the post hoc test results were evaluated, the RVECs and NF- $\kappa B$ levels of Group II were significantly higher than Group I, Group III, and Group IV ( $p<0.001$ for each). On the other hand, RVECs and NF- $\kappa B$ levels were similar between Group III, and Group IV ( $\mathrm{p}=0.58, \mathrm{p}=0.91$, respectively). It was observed that the RVECs values of Group III were higher than Group I and quite lower than Group II ( $<<0.001$ for each). Similarly, NF-kB levels of Group IV were found to be higher than Group I and significantly lower than Group II ( $p<0.001$ for each). 


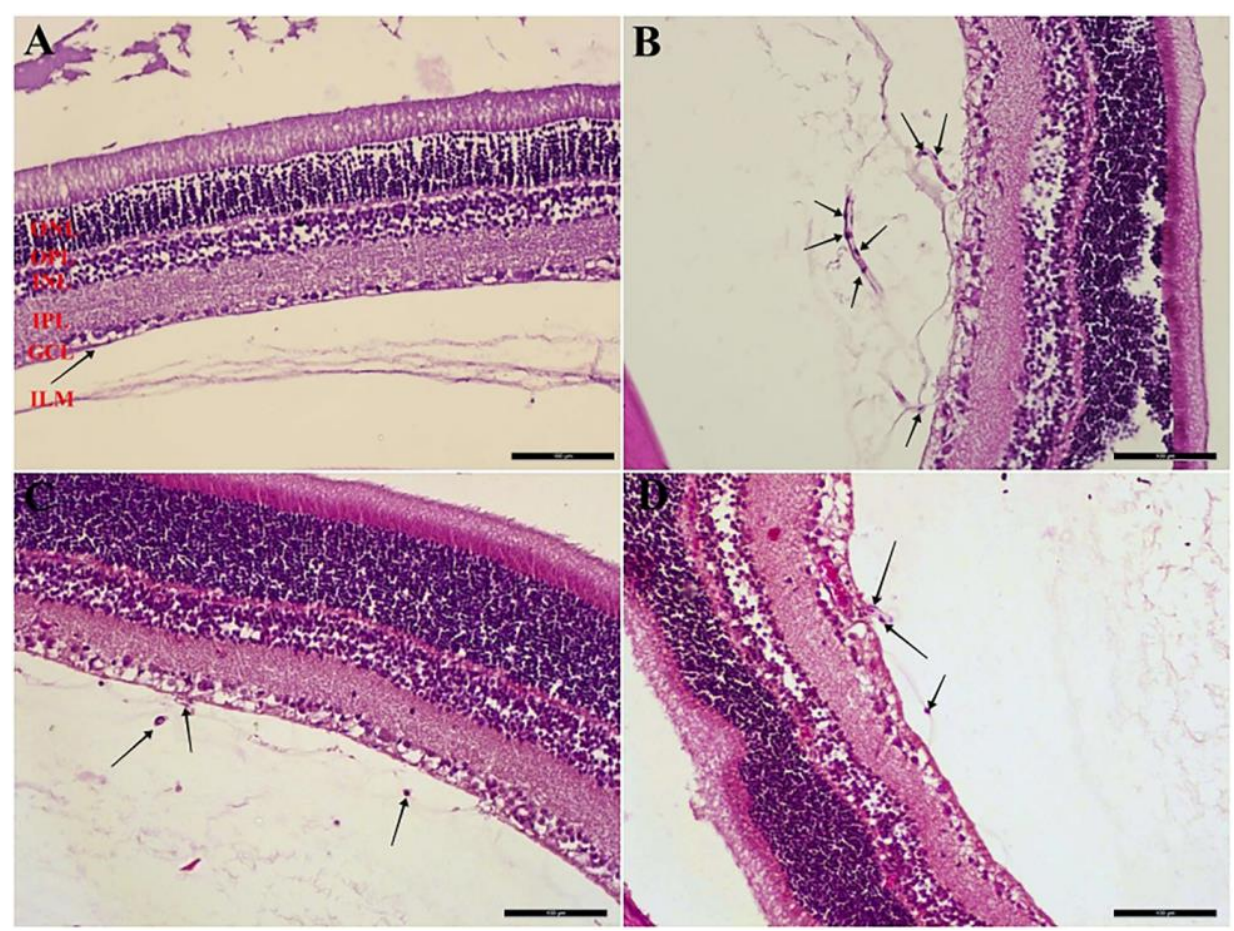

Figure 1. The representative retinal sections of retinal vascular endothelial cell nuclei (arrow) breaking through the ILM, stained with H\&E. A: Control group (Group I), B: Untreated OIR group (Group II), C: OIR group treated with bevacizumab and D: OIR group treated with carvacrol. ILM: Inner limiting membrane, GCL: Ganglion cell layer, IPL: Inner plexiform layer, INL: Inner nuclear layer, OIR: Oxygen-induced retinopathy, ONL: Outer nuclear layer, OPL: Outer plexiform layer.

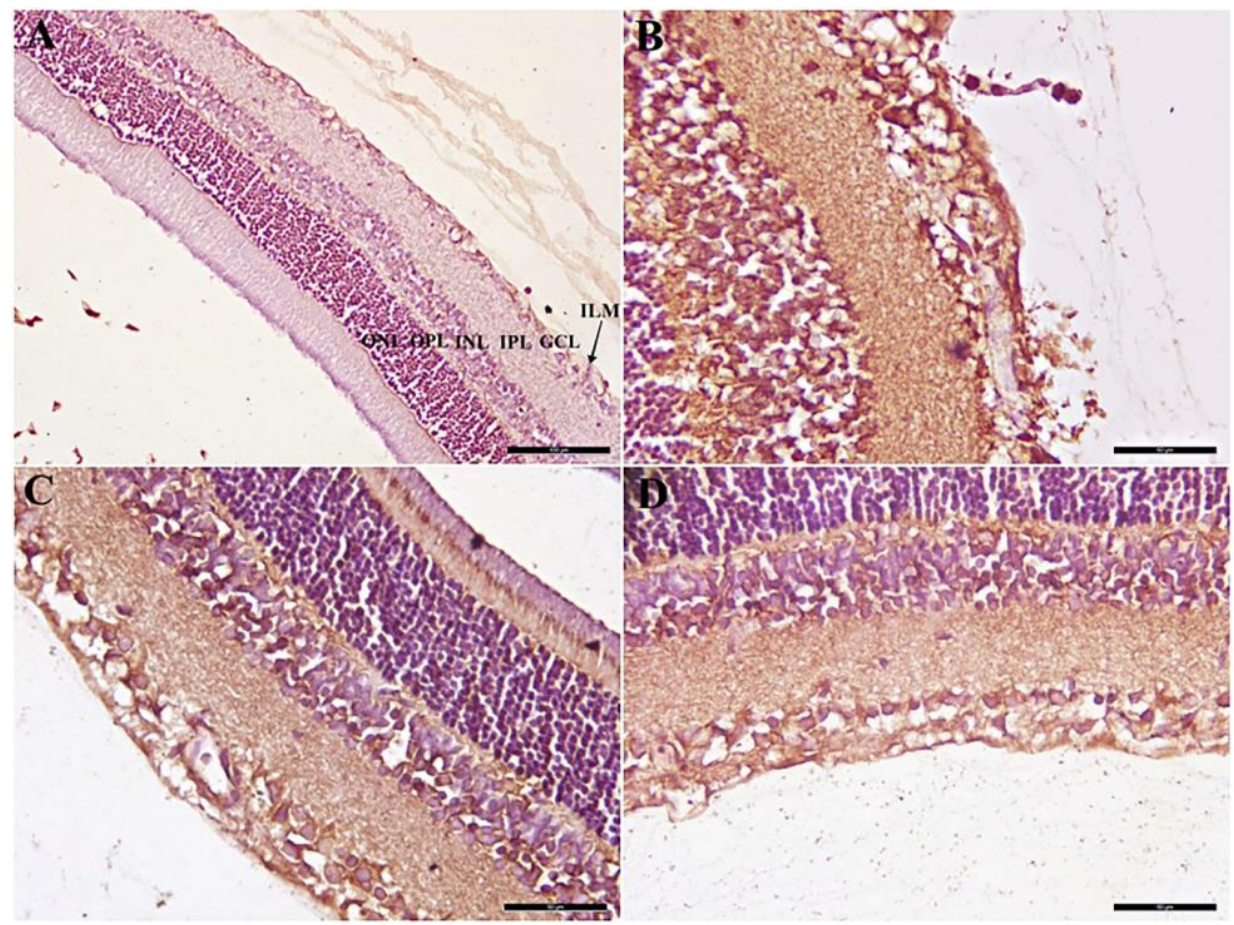

Figure 2. NF- $\kappa B$ immunohistochemical staining of the retina in the OIR model. A: Control group (Grade 0), B: Untreated OIR group (Grade 4), C: OIR group treated with bevacizumab (Grade 2) and D: OIR group treated with carvacrol (Grade 2). ILM: Inner limiting membrane, GCL: Ganglion cell layer, IPL: Inner plexiform layer, INL: Inner nuclear layer, OIR: Oxygen-induced retinopathy, ONL: Outer nuclear layer, OPL: Outer plexiform layer. 


\section{DISCUSSION}

The results in the present study showed that intraperitoneal carvacrol and bevacizumab treatment reversed the oxygen-dependent RVEC and NF- $\kappa \mathrm{B}$ elevation and suppressed angiogenesis in the OIR model. It is also important that the effect of carvacrol is similar to bevacizumab.

Bevacizumab is a powerful anti-angiogenic agent used in the treatment of colon cancer. It has found wide use in eye clinics after it has been found to suppress retinal NV. Carvacrol is a monoterpene with a phenolic structure (Baser, 1994). In addition to its anti-inflammatory and anti-oxidant properties, it has anti-angiogenic properties in cancer cases (Bayramoglu et al., 2014; Cui et al., 2015; Li et al., 2019; Mahmoodi et al., 2019). In many studies, it has been shown to suppress NV formation in different tissues and organs, but there is no study demonstrating its effect on retinal NV (Cui et al., 2015; Khan et al., 2019). In the present study, it was observed that carvacrol suppresses angiogenesis both directly and indirectly by affecting the formation of retinal NV.

The RVECs have an important role in angiogenesis. Ischemia and hypoxia in the retina lead to increased RVECs and production of angiogenic cytokines, thus leading to retinal NV formation (Cornel et al., 2015). Bevacizumab has been used in eye clinics for the treatment of pathological NV and macular edema for a long time (Maguire et al., 2016; Rich et al., 2006; Talks et al., 2016). Its anti-VEGF property is already known, but its effect on RVECs has not been studied. Likewise, the effect of carvacrol on RVECs is not known. In this study, it was found that both agents reduce RVECs in H\&E staining. Therefore, it can be said that both carvacrol and bevacizumab directly suppress $\mathrm{NV}$ formation at this step.

NF- $\kappa B$ suppresses VEGF by being released from RVECs and thus inhibits the formation of $\mathrm{NV}$ (Morais et al., 2009; Zhang et al., 2011). In studies other than eye diseases, carvacrol has been reported to suppress NF- $\kappa B$ (Aristatile et al., 2013; Kara et al., 2015). Likewise, Lopez et al. (2019) stated that bevacizumab suppresses NF- $\kappa \mathrm{B}$ in colorectal carcinoma (Lopez et al., 2019). However, the effect of carvacrol and bevacizumab on NF- $\kappa B$ in retinal tissues has not been reported in the literature. In the present study, as a result of retinal immunohistochemical staining, it was observed that both bevacizumab and carvacrol significantly decreased NF- $\kappa B$ and it was concluded that these two agents could also suppress angiogenesis indirectly.

There were some limitations in our study. Since the effective dose of intravitreal injection of carvacrol is not known and it is thought that intravitreal application in puppies may increase intraocular inflammation, the injections were administered as a single dose ip. Only RVECs and NF- $\kappa B$ were evaluated by $H \& E$ staining and semi-quantitative immunohistochemistry staining. Therefore, the parameters examined were limited and they were not supported by other advanced methods, such as Western blotting and real-time PCR. If antiinflammatory cytokines were also studied, the scope and power of the study could increase. Despite all these limitations, it is important to determine in our study that carvacrol and bevacizumab can inhibit angiogenesis directly and indirectly by suppressing RVECs and NF- $\mathrm{BB}$. In addition, it is important because it is the first study to reveal the effect of carvacrol on retinal NV and may guide more comprehensive studies on this subject.

\section{CONCLUSION}

In conclusion, carvacrol prevented pathological NV formation by suppressing RVECs and NF- $\kappa$ B in the experimental OIR model. In this respect, carvacrol can play an active role in the treatment of many eye diseases with pathological retinal NV formation. Therefore, further studies are needed in which carvacrol is studied in repeated doses intravitreally and more comprehensive parameters are evaluated.

\section{ACKNOWLEDGMENTS}

The authors thank Mr. Ayhan Çetinkaya for assistance with digital imaging.

Conflict of Interests: The authors declared that there is no conflict of interests.

Financial Disclosure: The authors declared that this study has received no financial support.

Author's Contributions: Concept: AK; Design: AK and SE; Literature search: AK; Experimental studies: AK; Immunohistochemical study and analysis: SE; Data acquisition: SE; Data analysis: SE; Statistical analysis: AK; Manuscript preparation: AK; Manuscript editing: AK; Manuscript review: AK and SE. 


\section{REFERENCES}

Acun G, Ozdemir H, Sunamak O, et al. The effect of single-dose intraperitoneal bevacizumab on peritoneal adhesion formation. Rev Invest Clin. 2018; 70(6):279-284.

Adamis AP, Miller JW, Bernal M-T, et al. Increased vascular endothelial growth factor levels in the vitreous of eyes with proliferative diabetic retinopathy. Am J Ophthalmol. 1994; 118(4):445-450.

Altundağ Ş, Aslım B. Kekiğin bazı bitki patojeni bakteriler üzerine antimikrobiyal etkisi. Orlab On-Line Mikrobiyoloji Dergisi. 2005; 3(7):12-24.

Aristatile B, Al-Assaf AH, Pugalendi KV. Carvacrol suppresses the expression of inflammatory marker genes in Dgalactosamine-hepatotoxic rats. Asian Pac J Trop Med. 2013; 6(3):205-211.

Ascaso FJ, Huerva V, Grzybowski A. The role of inflammation in the pathogenesis of macular edema secondary to retinal vascular diseases. Mediators Inflamm. 2014; 2014.

Baser K. Essential oils of Labiatae from Turkey: recent results. Lamiales Newsletter. 1994; 3:6-11.

Bayramoglu G, Senturk H, Bayramoglu A, et al. Carvacrol partially reverses symptoms of diabetes in STZ-induced diabetic rats. Cytotechnology. 2014; 66(2):251-257.

Cornel S, Adriana ID, Mihaela TC, et al. Anti-vascular endothelial growth factor indications in ocular disease. Rom J Ophthalmol. 2015; 59(4):235.

Cui Z-w, Xie Z-x, Wang B-f, et al. Carvacrol protects neuroblastoma SH-SY5Y cells against $\mathrm{Fe}$ 2+-induced apoptosis by suppressing activation of MAPK/JNK-NF- $\kappa \mathrm{B}$ signaling pathway. Acta Pharmacol Sin. 2015; 36(12):14261436.

Engerman RL. Pathogenesis of diabetic retinopathy. Diabetes. 1989; 38(10):1203-1206.

Ferris FL, Fine SL, Hyman L. Age-related macular degeneration and blindness due to neovascular maculopathy. Arch Ophtalmol. 1984; 102(11):1640-1642.

Gocmez SS, Yazir Y, Sahin D, Karadenizli S, Utkan T. The effect of a selective neuronal nitric oxide synthase inhibitor 3-bromo 7-nitroindazole on spatial learning and memory in rats. Pharmacol Biochem Behav. 2015; 131:19-25.

Green WR. Histopathology of age-related macular degeneration. Mol Vis. 1999; 5(27):1-10.

Halle M, Hall P, Tornvall P. Cardiovascular disease associated with radiotherapy: activation of nuclear factor kappa-B. J Intern Med. 2011; 269(5):469-477.

Hussein J, El-Bana M, Refaat E, El-Naggar ME. Synthesis of carvacrol-based nanoemulsion for treating neurodegenerative disorders in experimental diabetes. J Funct Foods. 2017; 37:441-448.

Kara M, Uslu S, Demirci F, Temel HE, Baydemir C. Supplemental carvacrol can reduce the severity of inflammation by influencing the production of mediators of inflammation. Inflammation. 2015; 38(3):1020-1027.

Khan I, Bhardwaj M, Shukla S, et al. Carvacrol encapsulated nanocarrier/nanoemulsion abrogates angiogenesis by downregulating COX-2, VEGF and CD31 in vitro and in vivo in a lung adenocarcinoma model. Colloids Surf B Biointerfaces. 2019; 181:612-622.
Klaassen I, Van Noorden CJ, Schlingemann RO. Molecular basis of the inner blood-retinal barrier and its breakdown in diabetic macular edema and other pathological conditions. Prog Retin Eye Res. 2013; 34:19-48.

Li Y, Xu Jz, Gu Cx, Liu Gl, Tian K. Carvacrol suppresses inflammatory responses in rheumatoid arthritis fibroblastlike synoviocytes. J Cell Biochem. 2019; 120(5):8169-8176.

Lopez A, Harada K, Vasilakopoulou M, Shanbhag N, Ajani JA. Targeting angiogenesis in colorectal carcinoma. Drugs. 2019; 79(1):63-74.

Maguire MG, Martin DF, Ying G-s, et al. Five-year outcomes with anti-vascular endothelial growth factor treatment of neovascular age-related macular degeneration: the comparison of age-related macular degeneration treatments trials. Ophthalmology. 2016; 123(8):1751-1761.

Mahmoodi M, Amiri H, Ayoobi F, et al. Carvacrol ameliorates experimental autoimmune encephalomyelitis through modulating pro-and anti-inflammatory cytokines. Life Sci. 2019; 219:257-263.

Morais C, Gobe G, Johnson DW, Healy H. Anti-angiogenic actions of pyrrolidine dithiocarbamate, a nuclear factor kappa B inhibitor. Angiogenesis 2009; 12(4):365.

Park K, Chen Y, Hu Y, et al. Nanoparticle-mediated expression of an angiogenic inhibitor ameliorates ischemia-induced retinal neovascularization and diabetes-induced retinal vascular leakage. Diabetes. 2009; 58(8):1902-1913.

Park SW, Kim JH, Kim KE, et al. Beta-lapachone inhibits pathological retinal neovascularization in oxygen-induced retinopathy via regulation of HIF-1 $\alpha$. J Cell Mol Med. 2014; 18(5):875-884.

Penn JS, Henry MM, Tolman BL. Exposure to alternating hypoxia and hyperoxia causes severe proliferative retinopathy in the newborn rat. Pediatr Res. 1994; 36(6):724731.

Rich RM, Rosenfeld PJ, Puliafito CA, et al. Short-term safety and efficacy of intravitreal bevacizumab (Avastin) for neovascular age-related macular degeneration. Retina. 2006; 26(5):495-511.

Smith L, Wesolowski E, McLellan A, et al. Oxygen-induced retinopathy in the mouse. Invest Ophthalmol Vis Sci. 1994; 35(1):101-111.

Şen HS, Şen V, Bozkurt M, et al. Carvacrol and pomegranate extract in treating methotrexate-induced lung oxidative injury in rats. Med Sci Monit. 2014; 20:1983.

Talks JS, Lotery AJ, Ghanchi F, et al. First-year visual acuity outcomes of providing aflibercept according to the VIEW study protocol for age-related macular degeneration. Ophthalmology. 2016; 123(2):337-343.

Zhang X-Z, Huang X, Qiao J-H, Zhang J-J, Zhang M-X. Inhibition of hypoxia-induced retinal neovascularization in mice with short hairpin RNA targeting Rac1, possibly via blockading redox signaling. Exp Eye Res. 2011; 92(6):473-481. 